\title{
Coherent Particle Transfer in an On-Demand Single-Electron Source
}

\author{
J. Keeling, ${ }^{1}$ A. V. Shytov, ${ }^{2}$ and L. S. Levitov ${ }^{3,4}$ \\ ${ }^{1}$ Cavendish Laboratory, University of Cambridge, Madingley Road, Cambridge CB3 OHE, United Kingdom \\ ${ }^{2}$ Department of Physics, University of Utah, Salt Lake City, Utah 84112, USA \\ ${ }^{3}$ Kavli Institute for Theoretical Physics, University of California Santa Barbara, California 93106, USA \\ ${ }^{4}$ Department of Physics, Massachusetts Institute of Technology, 77 Massachusetts Avenue, Cambridge, Massachusetts 02139, USA
}

(Received 27 April 2008; published 7 November 2008)

\begin{abstract}
Electron transfer from a localized state in a quantum dot into a ballistic conductor generally results in particle-hole excitations. We study this effect, considering a resonance level with time-dependent energy coupled to particle states in the Fermi sea. We find that, as the resonance level is driven through the Fermilevel, particle-hole excitations can be suppressed for certain driving protocols. In particular, such noiseless transfer occurs if the level moves with constant rapidity, its energy changing linearly with time. A scheme to study the coherence of particle transfer is proposed.
\end{abstract}

DOI: 10.1103/PhysRevLett.101.196404

Individual quantum states of light, supplied on demand by single-photon sources [1-3], are essential for current progress in manipulating and processing quantum information in quantum optics [4]. In particular, such sources are at the heart of secure transmission of quantum information by quantum cryptography [5], and of quantum teleportation [6]. An extension of these techniques to electron systems would be crucial for the inception of fermionbased quantum information processing $[7,8]$.

Elements of solid state electron optics, such as linear beam splitters $[9,10]$ and interferometers [11], have been known for a while, but an on-demand electron source was demonstrated only recently [12]. In the experiment [12] a localized state in a quantum dot, tunnel-coupled to a ballistic conductor (a quantum Hall edge channel), was charged and discharged by modulation of its energy induced by a periodic voltage on the gate, leading to a sequence of quantized single-electron current pulses [13].

Yet, the nearly perfect quantization of current pulses achieved in [12] does not guarantee full quantum coherence. In a fully coherent pulse, the injected electron occupies a prescribed quantum state without particle-hole pairs excited from the Fermi sea. However, as the particle or hole density of states is finite at low energy a generic perturbation applied to a Fermi system can create multiple pairs. This process is more disruptive than related process for photon sources (e.g., phonon emission), as these particlehole pairs occur in the same channel as the desired particle. Avoiding such excitations constrains the protocol for generating coherent pulses.

To study the coherence of particle transfer, we use an exact time-dependent scattering matrix, generalizing the Breit-Wigner theory of resonance scattering to arbitrary time dependence of the localized state energy $E(t)$. Applying this approach to the many-body evolution of a Fermi sea coupled to a localized state with driven energy, we find that for linear driving $E(t)=c t$, excitation creation is fully inhibited. The harmonic driving used in [12] is well
PACS numbers: 71.10.Pm, 03.65.Ud, 03.67.Hk, 73.50.Td

approximated by this linear model if electron release and capture occur well within each half-period, as shown in Fig. 1(a). For such clean protocols entanglement between the injected particle and the Fermi sea is totally suppressed by Pauli blocking of multiparticle excitations.

Clean protocols are not restricted to the adiabatic limit, and so one may study the form of clean current profiles as a function of speed of driving, which interpolates between Lorentzian when adiabatic, and exponential when fast, with fringes for intermediate rates. In the adiabatic limit, the results for the relevant states near the Fermi surface will match those for a time-dependent voltage pulse applied to the wire, Ref. [14]. We also study how robust such protocols are to imperfections expected in experiment, such as noise in the driving voltage. Our results can also be relevant for quantum pumps (see [15-17] and references therein).

A method to distinguish optimal and nonoptimal protocols is illustrated in Fig. 1(a), by measuring the shot noise from current partitioning on a beam splitter downstream of the electron source. For nonoptimal protocols, the total number of excitations $N^{\text {ex }}$ (electrons + holes) is greater
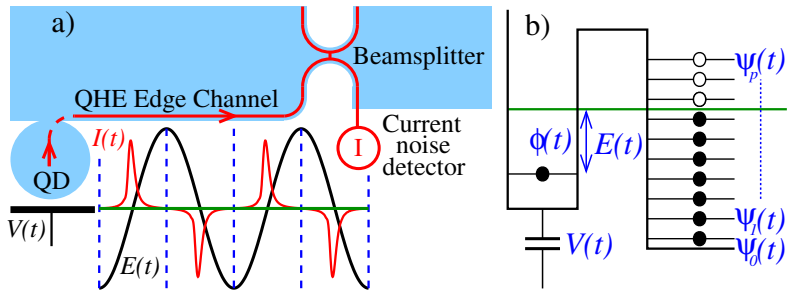

FIG. 1 (color online). (a) Quantum dot tunnel coupled to a ballistic conductor. Electrons are periodically trapped on the dot and injected in the conductor as the electron energy $E(t)$ in the dot is increased above the Fermi level when a time-dependent voltage $V(t)$ is applied to the gate. Particle-hole excitations accompanying the injected electron can be detected by the current partition noise on a beam splitter. (b) Schematic diagram of a localized level coupled to a continuum, Eq. (1). 
than one. Because every electronic state scatters independently, the variance of transferred charge depends on the number of excitations that may scatter. Hence, the DC shot noise generated on the beam splitter is $e^{2} \mathrm{t}(1-\mathrm{t}) N^{\mathrm{ex}} \nu$, where $t$ is the beam splitter transmission coefficient and $\nu$ is the frequency of current pulses.

In the setup of Ref. [12] the gate used to vary $E(t)$ is placed so close to the dot that the charging energy $e^{2} / 2 C$ is small compared to level spacing, which allows us to leave out the Hubbard-like interaction term. Also, because a magnetic field of a few Tesla was applied to create a quantum Hall state in which electron spins are polarized, only one spin projection is considered; hence, electron transfer from a quantum dot to the Fermi sea is described by the many-body Hamiltonian [Fig. 1(b)]:

$$
H=E(t) d^{\dagger} d+\sum_{p} \varepsilon_{p} a_{p}^{\dagger} a_{p}+\lambda_{p}(t) d^{\dagger} a_{p}+\lambda_{p}^{*}(t) a_{p}^{\dagger} d
$$

where $d$ and $a_{p}$ describe localized and extended states. Here, the electron energy in the dot $E(t)$ and tunnelling amplitude $\lambda(t)$ are both taken as time dependent.

Crucially, because the localized state is coupled to the continuum at all times, its behavior (e.g., charging or discharging) can be fully accounted for by an $S$ matrix for transitions in the continuum. The situation here is completely analogous to the Breit-Wigner theory of resonance scattering in which an energy-dependent scattering phase is used to describe the resonance. To find this $S$ matrix, we must solve first the single-particle problem, which is a generalization of the Friedrichs model [18] to timedependent energy of the localized level [19]. With this aim, we begin by solving the Schrödinger equations for the propagating modes $\psi_{p}(t)$ coupled to the wavefunction $\varphi(t)$ of the localized state:

$\left[i \partial_{t}-\varepsilon_{p}\right] \psi_{p}=\lambda_{p}^{*}(t) \varphi, \quad\left[i \partial_{t}-E(t)\right] \varphi=\sum_{p} \lambda_{p}(t) \psi_{p}$

(we set $\hbar=1$ and $\varepsilon_{\mathrm{F}}=0$ unless specified otherwise).

Because the continuum of propagating modes in [12] is one dimensional, it is convenient to go over to position representation $\psi(t, x)=\sum_{p} e^{i p x} \psi_{p}(t)$. Hereafter we assume a constant density of states and treat the couplings $\lambda_{p}$ as energy independent. Replacing $\varepsilon_{p}$ by $-i v_{\mathrm{F}} \partial_{x}$, where $v_{\mathrm{F}}$ is the Fermi velocity, gives

$$
\begin{gathered}
{\left[i \partial_{t}-E(t)\right] \varphi(t)=\lambda(t) \int d x \delta(x) \psi(t, x),} \\
{\left[i \partial_{t}+i v_{\mathrm{F}} \partial_{x}\right] \psi(t, x)=\lambda^{*}(t) \delta(x) \varphi(t) .}
\end{gathered}
$$

The scattering matrix for energy-nonconserving time evolution can be labeled by pairs of energies of the continuum states, as $U\left(\varepsilon, \varepsilon^{\prime}\right)$. For $x<0$ continuum modes propagate freely and the initial state is $\psi(t, x<0)=\psi_{0}(t, x)=$ $e^{-i \varepsilon^{\prime} t}$, with $\tilde{t}=t-x / v_{\mathrm{F}}$, and $\varphi(t=-\infty)=0$ as discussed above. Projecting the evolved state onto a corresponding final state gives

$$
U\left(\varepsilon, \varepsilon^{\prime}\right)=\int d t \psi(t, x>0) e^{i \varepsilon \tilde{t}} .
$$

Let us now solve the coupled equations of motion, and thus find $U\left(\varepsilon, \varepsilon^{\prime}\right)$. First solving Eq. (3), one finds

$$
\psi(t, x)=\psi_{0}(\tilde{t})-\frac{i}{v_{\mathrm{F}}} \lambda^{*}(\tilde{t}) \varphi(\tilde{t}) \theta(x) .
$$

Substituting this into Eq. (2) we find an equation for the localized state: $\left[i \partial_{t}-E(t)+i \gamma(t) / 2\right] \varphi(t)=\lambda(t) \psi_{0}(t)$, where we introduced notation $\gamma(t)=|\lambda(t)|^{2} / v_{\mathrm{F}}$ for the localized level linewidth. Then, the solution of this equation with the initial condition $\varphi(-\infty)=0$ is of the form

$$
\varphi(t)=-i \int_{-\infty}^{t} d t^{\prime} \lambda\left(t^{\prime}\right) \psi_{0}\left(t^{\prime}\right) e^{X\left(t, t^{\prime}\right)},
$$

where $X\left(t, t^{\prime}\right)=-\int_{t^{\prime}}^{t}\left[\frac{1}{2} \gamma(\tau)+i E(\tau)\right] d \tau$. The result (6) may be substituted into Eq. (5) for $\psi(t, x)$; this can in turn be used in Eq. (4) to evaluate $U\left(\varepsilon, \varepsilon^{\prime}\right)$. Putting all this together, we find the $S$ matrix

$$
\begin{gathered}
U\left(\varepsilon, \varepsilon^{\prime}\right)=\iint d t d t^{\prime} e^{i \varepsilon t-i \varepsilon^{\prime} t^{\prime}} U\left(t, t^{\prime}\right), \\
U\left(t, t^{\prime}\right)=\delta\left(t-t^{\prime}\right)-\theta\left(t-t^{\prime}\right) \frac{\lambda^{*}(t) \lambda\left(t^{\prime}\right)}{v_{\mathrm{F}}} e^{X\left(t, t^{\prime}\right)} .
\end{gathered}
$$

It is straightforward to show that $U$ is unitary, $U^{\dagger} U=\hat{1}$, by verifying that $\int U(\tau, t) U^{*}\left(\tau, t^{\prime}\right) d \tau=\delta\left(t-t^{\prime}\right)$.

As a sanity check, let us apply these results to a stationary level. For time-independent $\gamma$ and $E$, we find $X\left(t, t^{\prime}\right)=-\left(\frac{\gamma}{2}+i E\right)\left(t-t^{\prime}\right)$. Integrating over $t$ and $t^{\prime}$ in (7), we obtain the familiar result

$$
U\left(\varepsilon, \varepsilon^{\prime}\right)=2 \pi \delta\left(\varepsilon-\varepsilon^{\prime}\right) \frac{\varepsilon-E-i \gamma / 2}{\varepsilon-E+i \gamma / 2} .
$$

A more interesting example is a level moving at a constant rapidity, $E(t)=c t$. In this case, $X\left(t, t^{\prime}\right)=-\frac{\gamma}{2}\left(t-t^{\prime}\right)-$ $\frac{i c}{2}\left(t^{2}-t^{\prime 2}\right)$. After integrating over $t$ and $t^{\prime}$ in (7) we find $U\left(\varepsilon, \varepsilon^{\prime}\right)=2 \pi \delta\left(\varepsilon-\varepsilon^{\prime}\right)+T\left(\varepsilon, \varepsilon^{\prime}\right)$ where

$$
T\left(\varepsilon, \varepsilon^{\prime}\right)=-2 \pi \frac{\gamma}{|c|} \theta\left(\varepsilon-\varepsilon^{\prime}\right) e^{-(\gamma / 2 c)\left(\varepsilon-\varepsilon^{\prime}\right)+(i / 2 c)\left(\varepsilon^{2}-\varepsilon^{\prime 2}\right)}
$$

for $c>0$, and with $\theta\left(\varepsilon^{\prime}-\varepsilon\right)$ instead of $\theta\left(\varepsilon-\varepsilon^{\prime}\right)$ for $c<$ 0 . This result agrees with the continuum limit of the Demkov-Osherov $S$ matrix [20,21] for a single level crossing a group of stationary levels.

We next employ the single-particle $S$ matrix (7) in the calculation of the many-body properties, taking as the initial state the filled Fermi sea. The number of excitations can be obtained from the filled Fermi sea state $|\Omega\rangle$ evolved with the $S$ matrix $U\left(\varepsilon, \varepsilon^{\prime}\right)$. In particular, the number of fermions promoted above the Fermi level is

$$
N^{+}=\left\langle\Omega\left|U^{\dagger} \sum_{\varepsilon>0} a_{\varepsilon}^{\dagger} a_{\varepsilon} U\right| \Omega\right\rangle=\sum_{\varepsilon>0, \varepsilon^{\prime}<0}\left|U\left(\varepsilon, \varepsilon^{\prime}\right)\right|^{2} .
$$

Similarly, the number of holes created below the Fermi surface $N^{-}$is found by swapping $\varepsilon$ and $\varepsilon^{\prime}$ in Eq. (10).

Using Eq. (7), our explicit expression for $U\left(\varepsilon, \varepsilon^{\prime}\right)$, and considering a general time-dependence $E(t)$, but assuming 
now $\gamma(t)=\gamma$ for simplicity, one may rewrite the result (10) by using $\int_{0}^{\infty} d \varepsilon e^{i \varepsilon(t-s)}=\frac{i}{t-s+i 0}$, which yields

$$
N^{ \pm}=-\left(\frac{\gamma}{2 \pi}\right)^{2} \iiint \int_{t>t^{\prime}, s>s^{\prime}} \frac{e^{X\left(t, t^{\prime}\right)+X^{*}\left(s, s^{\prime}\right)} d t d t^{\prime} d s d s^{\prime}}{(t-s \pm i 0)\left(t^{\prime}-s^{\prime} \pm i 0\right)} .
$$

It can be seen from these expressions that the numbers of excited particles and holes are not constrained.

To illustrate this, let us first consider a highly nonoptimal protocol for $E(t)$, where the level first moves rapidly to the Fermi-level, remains there for time $\Delta t \gg \gamma^{-1}$, and then moves rapidly away. During the time $\Delta t$, the level acts as a resonant perturbation for the Fermi sea, with scattering phase $\delta(\varepsilon)=\tan ^{-1} \frac{2(\varepsilon-E)}{\gamma}-\frac{\pi}{2}$ defined by Eq. (8). This creates a logarithmically divergent number of excitations, $N^{ \pm} \propto \log \Delta t$, which can be viewed as an example of the "orthogonality catastrophe" [22].

The situation is completely different in the case when the level moves linearly, $E(t)=c t$. From our result for the $S$ matrix, Eq. (9), we have $U\left(\varepsilon, \varepsilon^{\prime}>\varepsilon\right)=0$ for $c>0$. This means that no holes are excited when the level is moving up in energy: $N^{-}=0$ for $c>0$. (Similarly when the level is moving down, i.e., $c<0$, one has $U\left(\varepsilon, \varepsilon^{\prime}<\varepsilon\right)=0$ and thus $\left.N^{+}=0\right)$. At the same time, we expect that $N^{+}-$ $N^{-}=+1(-1)$ when the level moves up (down) as just one particle is transferred between the localized level and continuum. Indeed, we can find $N^{ \pm}$directly, by substituting $U$ into Eq. (10) yielding $N^{+}=1$ for $c>0$ (and $N^{-}=1$ for $c<0$ ). Thus for linear driving, a single fermion is coherently transferred from the localized level to the continuum, and no holes are created.

This remarkable behavior can also be understood directly from Eq. (9): restricted to $\varepsilon>0, \varepsilon^{\prime}<0, T\left(\varepsilon, \varepsilon^{\prime}\right)$ is a rank one matrix. As discussed in Ref. [14], for a rank one $S$ matrix the exact many-body state is a product of an unperturbed Fermi sea and an extra particle occupying one mode, which is a superposition of harmonics with $\varepsilon>$ 0 . The latter can be read off directly from (9):

$$
\psi(t, x)=\sqrt{\frac{\gamma}{2 \pi c}} \int_{0}^{\infty} d \varepsilon \exp \left[-i \varepsilon \tilde{t}-\frac{\gamma \varepsilon}{2 c}+i \frac{\varepsilon^{2}}{2 c}\right] .
$$

This gives a density profile $|\psi(x, t)|^{2}$ that is the convolution of a Lorentzian, width $\gamma / c$, with a Fresnel integral, leading to fringes on the trailing side of the pulse (Fig. 2). In the limit $|d E / d t| \ll \gamma^{2}$, the behavior of the relevant levels near the Fermi surface $[|\epsilon| \ll \min (E, \gamma)]$ is equivalent to applying a voltage pulse to a one dimensional wire as in Ref. [14], with $e V(t)=2 d \theta / d t ; \tan (\theta(t))=2 E(t) / \gamma$.

Under periodic driving, as used in Ref. [12], the same key features of particle transfer as presented here can be reproduced, as long as the driving is effectively linear near the Fermi surface. In particular, if the period is long compared to $\max \left(\gamma^{-1}, \gamma / c\right)$, and the extremal value of $E(t)$ exceeds $\gamma$, as indicated in Fig. 1(a); then particle transfer will be nearly noiseless, close to that under linear driving. From Eq. (12), one sees the current pulse involves

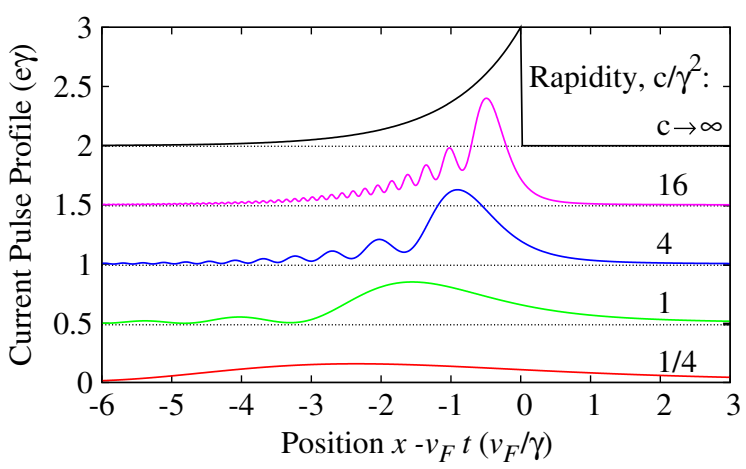

FIG. 2 (color online). Single-electron current pulse produced by linear driving of a localized state of width $\gamma$ across the Fermi level, $E(t)=c t$. The pulse profile $|\psi(t, x)|^{2}$, Eq. (12), for $x>0$, is shown for different values of rapidity $c$. Different curves are offset by $0.5 e \gamma$. The asymptotic form is Lorentzian at slow driving, and exponential at fast driving. Note interference fringes at the trailing side of the pulse at $c \gtrsim \gamma^{2}$.

energies up to $\varepsilon \simeq c / \gamma$, so for the profile to match Fig. 2 then the electron dispersion must be linear up to this energy. However, comparison to Ref. [20] indicates that particle transfer will remain noiseless in a more general case, when the tunnel coupling and the density of states in Eq. (1) are energy dependent. An extra requirement to study these coherent electron pulses is that the temperature of the Fermi sea is much less than $\min (\gamma, c / \gamma)$, to allow noise due to the current pulse to be distinguished from background thermal noise.

More insight into the robustness of the coherent particle transfer can be gained by considering, as an example, the effect of classical noise added to $E(t)$ (for a discussion of Landau-Zener transitions in the presence of different kinds of noise see [23-25] and references therein). In experimental realizations, the energy of the localized level is not under perfect control; as well as the desired applied voltage, there will be Johnson noise and noise associated with fluctuating charges. For simplicity we consider the effect of noise on the linear driving case [see Fig. 3(a)]:

$$
E(t)=c t+\xi(t)
$$

where $\langle\xi(t)\rangle=0$ and $\left\langle\xi(t) \xi\left(t^{\prime}\right)\right\rangle=\gamma_{2} \delta\left(t-t^{\prime}\right)$. Substituting this into the equation for number of excitations, Eq. (11), and averaging over realizations of the noise, we find that the integrand can be written as
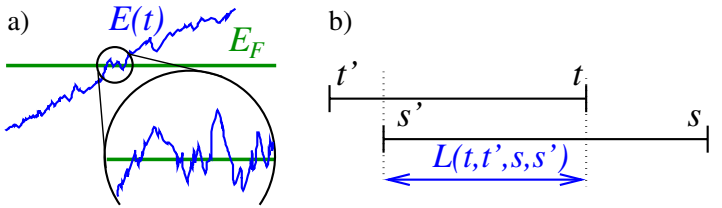

FIG. 3 (color online). (a) Multiple crossing of the Fermi level in the presence of noise, Eq. (13), leads to creation of particle or hole excitations; (b) Overlap of time intervals and definition of $L\left(t, t^{\prime}, s, s^{\prime}\right)$. 


$$
\frac{e^{-(\gamma / 2)\left(t-t^{\prime}+s-s^{\prime}\right)-(i c / 2)\left(t^{2}-t^{\prime 2}-s^{2}+s^{\prime 2}\right)}}{(t-s+i 0)\left(t^{\prime}-s^{\prime}+i 0\right)} F\left(t, t^{\prime}, s, s^{\prime}\right),
$$

where the factor $F=\left\langle e^{i \int_{s^{\prime}}^{s} \xi(\tau) d \tau-i \int_{t^{\prime}}^{t} \xi(\tau) d \tau}\right\rangle$ equals

$$
\exp \left(-\frac{\gamma_{2}}{2}\left[\left|t-t^{\prime}\right|+\left|s-s^{\prime}\right|-2 L\left(t, t^{\prime}, s, s^{\prime}\right)\right]\right) .
$$

Here $L\left(t, t^{\prime}, s, s^{\prime}\right)$ is the overlap between the two intervals $\left[t, t^{\prime}\right]$ and $\left[s, s^{\prime}\right]$ [see Fig. 3(b)]. This simple form (15) comes from the Gaussian correlations of $\xi(\tau)$ leading to cancellation for any region inside $L\left(t, t^{\prime}, s, s^{\prime}\right)$.

To make further progress, we change variables to $s=$ $t+\eta, t^{\prime}=t-\Delta_{t}, s^{\prime}=s-\Delta_{s}$. Because $F$ does not depend on central time $t$, the only $t$ dependence in (14) comes from $t^{2}-t^{\prime 2}-s^{2}+s^{\prime 2}=\Delta_{t}\left(2 t-\Delta_{t}\right)-\Delta_{s}(2 t+2 \eta-$ $\Delta_{s}$ ). Integration over $t$ thus gives a delta function, $\frac{2 \pi}{c} \delta\left(\Delta_{t}-\Delta_{s}\right)$, which considerably simplifies the expression:

$$
N^{ \pm}=\frac{-\gamma^{2}}{2 \pi c} \int_{-\infty}^{\infty} d \eta \int_{0}^{\infty} d u \frac{e^{(-i c \eta-\gamma) u+\gamma_{2}(L(\eta, u)-u)}}{(\eta \pm i 0)^{2}},
$$

where $u=\Delta_{t}=\Delta_{s}$, and the overlap can now be written $L(\eta, u)=\theta(u-|\eta|)(u-|\eta|)=u-\min (u,|\eta|)$.

Let us now examine the asymptotic limits of expression (16); it is convenient to consider the total number of excitations $N^{\mathrm{ex}}=N^{+}+N^{-}$. [Because of fermion conservation, for an initially populated localized state, $N^{+}-$ $N^{-}=1$.] Taking $N^{ \pm}$from Eq. (16) we use the identity $(\eta+i 0)^{-2}+(\eta-i 0)^{-2}=-\int_{-\infty}^{\infty} d \omega|\omega| e^{i \eta \omega}$ and, after relabeling $\omega$ to $\omega+c u$ and with $\tilde{u}=u+\omega / c$, obtain

$N^{\mathrm{ex}}=\frac{\gamma^{2}}{2 \pi} \int_{-\infty}^{\infty} d \omega \int_{-\infty}^{\infty} d \eta \int_{0}^{\infty} d u|\tilde{u}| e^{i \omega \eta-\gamma u-\gamma_{2} \min (u,|\eta|)}$.

In this form, it is easy to extract the asymptotic limits of fast and slow driving. At large $c$, we may approximate $\mid u+$ $\omega / c \mid \approx u$. Then, integration over $\omega$ yields $2 \pi \delta(\eta)$, giving $\lim _{c \rightarrow \infty} N^{\mathrm{ex}}=1$. This limit has a simple interpretation; if driven fast enough, the effects of noise do not matter, and one recovers the clean case discussed earlier.

In the limit of small $c$, we retain only the terms proportional to $1 / c$. Defining $\gamma_{*}=\gamma+\gamma_{2}$, we may write

$$
\int_{0}^{\infty} d u e^{-\gamma u-\gamma_{2} \min (u,|\eta|)}=\frac{1}{\gamma_{*}}+\frac{\gamma_{2}}{\gamma \gamma_{*}} e^{-\gamma^{*}|\eta|} .
$$

The integration over $\eta$ then gives

$$
N^{\mathrm{ex}}=\frac{2 \gamma \gamma_{2}}{\pi c} \int_{0}^{\infty} d \omega \frac{\omega}{\omega^{2}+\gamma_{*}^{2}} \approx \frac{2 \gamma \gamma_{2}}{\pi c} \ln \frac{\omega_{0}}{\gamma_{*}} .
$$

In the final expression, we have introduced a high $\omega$ cutoff $\omega_{0}$; this corresponds to replacing the white noise $\delta\left(t-t^{\prime}\right)$ correlations of $\xi(t)$ with a correlation function having a short time cutoff $1 / \omega_{0}$. This replacement is necessary because for a truly white spectrum, there will be an infinite number of crossings of the Fermi level, and so an infinite number of excitations. By comparing the fast and slow driving limits, we find the crossover occurs at the rapidity $c=(2 / \pi) \gamma \gamma_{2} \ln \left(\omega_{0} / \gamma_{*}\right)$.

In conclusion, excitation of particle-hole pairs in a single-electron source can be suppressed by optimizing the protocol of particle transfer between a localized state and continuum. The transfer is totally noiseless when the energy of the localized state varies linearly in time. In this case, owing to the Fermi statistics, particle-hole pair production is suppressed by Pauli blocking of multiparticle excitations. The quantum state resulting from such clean transfer is a product state of a particle added to an unperturbed Fermi sea, with zero entanglement between them. Particle or hole excitation, and its suppression, can be observed directly by noise measurement.

We are grateful to Christian Glattli and Israel Klich for useful discussion. J.K. acknowledges financial support from the Lindemann Trust, and Pembroke College Cambridge. L.L.'s work was partially supported by the W. M. Keck Foundation Center for Extreme Quantum Information Theory and by the NSF Grant No. PHY0551164.

[1] A. Imamoḡlu and Y. Yamamoto, Phys. Rev. Lett. 72, 210 (1994).

[2] C. Brunel, B. Lounis, Ph. Tamarat, and M. Orrit, Phys. Rev. Lett. 83, 2722 (1999).

[3] N. Akopian et al., Phys. Rev. Lett. 96, 130501 (2006).

[4] P. Kok et al., Rev. Mod. Phys. 79, 135 (2007).

[5] C. H. Bennett et al., J. Cryptology 5, 3 (1992).

[6] D. Bouwmeester et al., Nature (London) 390, 575 (1997).

[7] C. W. J. Beenakker, C. Emary, M. Kindermann, and J.L. van Velsen, Phys. Rev. Lett. 91, 147901 (2003).

[8] P. Samuelsson, E. V. Sukhorukov, and M. Büttiker, Phys. Rev. Lett. 92, 026805 (2004).

[9] B. J. van Wees et al., Phys. Rev. Lett. 60, 848 (1988).

[10] D. A. Wharam et al., J. Phys. C 21, L209 (1988).

[11] Y. Ji et al., Nature (London) 422, 415 (2003).

[12] G. Fève et al., Science 316, 1169 (2007).

[13] M. Moskalets, P. Samuelsson, and M. Büttiker, Phys. Rev. Lett. 100, 086601 (2008).

[14] J. Keeling, I. Klich, and L. S. Levitov, Phys. Rev. Lett. 97, 116403 (2006).

[15] L. J. Geerligs et al., Phys. Rev. Lett. 64, 2691 (1990).

[16] M. D. Blumenthal et al., Nature Phys. 3, 343 (2007)

[17] M. Moskalets and M. Büttiker, Phys. Rev. B 66, 035306 (2002); Phys. Rev. B 66, 205320 (2002).

[18] K. O. Friedrichs, Commun. Pure Appl. Math. 1, 361 (1948).

[19] V. V. Kocharovsky et al., Comput. Math. Appl. 34, 727 (1997).

[20] Y. N. Demkov and V. I. Osherov, Zh. Eksp. Teor. Fiz. 53, 1589 (1967) [Sov. Phys. JETP 26, 916 (1968)].

[21] N. A. Sinitsyn, Phys. Rev. B 66, 205303 (2002).

[22] P. W. Anderson, Phys. Rev. Lett. 18, 1049 (1967).

[23] Y. Kayanuma and H. Nakayama, Phys. Rev. B 57, 13099 (1998).

[24] V.L. Pokrovsky and N.A. Sinitsyn, Phys. Rev. B 67, 144303 (2003).

[25] M. Wubs et al., Phys. Rev. Lett. 97, 200404 (2006). 